\title{
Definition of Saturn's magnetospheric model parameters for the Pioneer 11 flyby
}

\author{
E. S. Belenkaya, I. I. Alexeev, V. V. Kalegaev, and M. S. Blokhina \\ Skobeltsyn Institute of Nuclear Physics, Moscow State University, Moscow 119992 Russia
}

Received: 29 November 2005 - Revised: 12 February 2006 - Accepted: 13 March 2006 - Published: 19 May 2006

\begin{abstract}
This paper presents a description of a method for selection parameters for a global paraboloid model of Saturn's magnetosphere. The model is based on the preexisting paraboloid terrestrial and Jovian models of the magnetospheric field. Interaction of the solar wind with the magnetosphere, i.e. the magnetotail current system, and the magnetopause currents screening all magnetospheric field sources, is taken into account. The input model parameters are determined from observations of the Pioneer 11 inbound flyby.
\end{abstract}

Keywords. Magnetospheric physics (Current systems; Magnetospheric configuration and dynamics; Planetary magnetospheres)

\section{Introduction}

Pioneer 11, launched on 15 April 1973, was the first spacecraft which encountered Saturn. It reached the point of closest approach (CA) to Saturn on 1 September 1979. Voyager 1 and 2 were the next spacecraft to pass through Saturn's magnetosphere. Voyager 1 reached the point of closest approach to Saturn on 12 November 1980, and Voyager 2 on $26 \mathrm{Au}-$ gust 1981. Spacecraft Cassini was launched in 1997 to Saturn, with its approach on 1 July 2004, and became a Saturn orbiter. Pioneer 11 and Voyager 1 and 2 entered Saturn's magnetopause near the local noon and exited on the dawn side (Voyager 2 and Pioneer 11 near the dawn meridian, and Voyager 1 further down the tail at $\sim 03: 30$ LT). Cassini will orbit Saturn for 4 years and use gravitational assistance from Titan to change its orbit inclination and orientation.

Saturn is a rapidly rotating planet (the angular velocity of Saturn is $\Omega_{s}=1.638 \cdot 10^{-4} \mathrm{~s}^{-1}$ ), possessing a strong magnetic field: the dipole magnetic moment of Saturn, $\mathbf{M}_{S}$, equals

Correspondence to: E. S. Belenkaya

(elena@dec1.sinp.msu.ru)
$4.6 \cdot 10^{13} \mathrm{G} \cdot \mathrm{km}^{3}$ or $0.2 \mathrm{G} \cdot R_{S}{ }^{3}$ and is directed north, opposite to that of the Earth but the same as Jupiter's field; the average Saturn radius is $R_{S}=60330 \mathrm{~km}$. Saturn's internal magnetic field is closely symmetric about the spin axis of the planet, and can be modeled as the sum of the axisymmetric dipole, quadrupole and octupole terms. The octupole terms for Saturn are considerably smaller than for Earth and Jupiter (Smith et al., 1980). The vector dipole moment has a tilt angle of less than $1^{\circ}$ (for the Earth and Jupiter the tilt angle is about $10^{\circ}$ ) and is slightly offset along the polar axis $\left(0.04 \pm 0.02 R_{S}\right.$ (Smith et al., 1980)). The SPV (Saturn Pioneer Voyager) model (Davis and Smith, 1990) value of the ionospheric field strength at Saturn's equator is $B_{S 0}=21160 \mathrm{nT}$.

Inside $10 R_{S}$ the magnetic field observed by Pioneer 11 was predominantly that of the planetary dipole; beyond $10 R_{S}$ near the noon meridian, the field topology was characteristic of a dipole field being compressed by a highspeed solar wind (Smith et al., 1980). The subsolar magnetopause distance for the kronian magnetosphere, $R_{s s}$, (according to data before Cassini) is $\sim 17-24 R_{S}$ (Behannon et al., 1983; Maurice and Engel, 1995). During the Pioneer 11 approach to Saturn, a fast solar wind stream with velocity $V_{s w} \sim 470 \mathrm{~km} / \mathrm{s}$ and magnetic field $B_{I M F} \leq 1 \mathrm{nT}$ passed the spacecraft (Maclennan et al., 1983) and compressed the dayside magnetopause up to $R_{S S} \sim 17 R_{S}$ (Smith et al., 1980), while, for example, for Voyager $1, R_{s s}$ was $23-24 R_{S}$ (Connerney et al., 1983; Maclennan et al., 1983), and for Voyager 2, $\sim 19 R_{S}$ (Behannon et al., 1983). The solar wind conditions were relatively quiet during the Voyager 1 encounter and disturbed during the Voyager 2 approach to Saturn (Behannon et al., 1983).

The plasma motions in Saturn's magnetosphere are driven by planetary rotation and the solar wind interaction (e.g. Cowley et al., 2004). Voyager 1 found the tailward flowing plasma near the pre-dawn magnetopause, a phenomenon well-known for the Earth and Jupiter. This was not ob-

Published by Copernicus GmbH on behalf of the European Geosciences Union. 
served by Voyager 2 at Saturn, probably because Saturn was immersed in Jupiter's magnetotail during the time of the Voyager 2 encounter period, since the plasma flux in the Jovian tail is lower than that in the solar wind (Behannon et al., 1983).

As noted in the work of Smith et al. (1980), the corotation electric field can dominate the convective electric field due to the solar wind, as far out to radial distances in excess of $21 R_{S}$ (the average radial position of the noon magnetopause boundary). The plasmas in the kronian magnetosphere rigidly corotate roughly out to $10 R_{S}$ (the breakdown in rigid corotation is outside of $\sim 12.5 R_{S}$ for Pioneer 11 (Bastian et al., 1980), $\sim 4.5 R_{S}$ for Voyager 1 , and $\sim 6.5 R_{S}$ for Voyager 2 (Richardson, 1986)); beyond $10 R_{S}$ the partial corotation with a speed $\sim 0.3-0.8$ of the rigid corotation speed exists (Frank et al., 1980). According to Voyager 2 data, the plasma angular velocity decreases from near-rigid corotation in the inner magnetosphere, to half of the rigid corotation at $\sim 15-20 R_{S}$ in the equatorial plane (Richardson, 1986), which corresponds to $\sim 13^{\circ}-15^{\circ}$ co-latitude in the northern ionosphere and $\sim 14^{\circ}-17^{\circ}$ in the southern ionosphere (Cowley and Bunce, 2003). For the Voyager 1 encounter the plasma subcorotated throughout the magnetosphere (Richardson, 1986). (In the terrestrial magnetosphere, corotation is stopped at $\sim 6 R_{E}$.)

A condition determining plasma outflow is requiring the rotation speed, $V_{\varphi}$, to exceed the local Alfvén speed, $V_{A}$. This happened at the distance which is called the Alfvén radius, $r_{A}$. For Saturn $r_{A}$ could be estimated approximately as 6-8 $R_{S}$ (Frank et al., 1980).

Cowley et al. (2004) noted that the outermost magnetospheric region driven by the interaction with the solar wind is dominated by reconnection between the southward directed planetary field in the equatorial magnetosphere and a northward pointing interplanetary magnetic field (IMF). The solar wind electric field is $\mathbf{E}_{I E F}=-\left[\mathbf{V}_{s w} \times \mathbf{B}_{I M F}\right]$. On the average, the solar wind speed, $\mathbf{V}_{s w}$, is approximately constant with heliocentric distance, while the interplanetary magnetic field (IMF), $\mathbf{B}_{I M F}$, varies inversely with distance. Thus, at Saturn interplanetary fields $\mathbf{B}_{I M F}$ and $\mathbf{E}_{I E F}$ should be 10 times weaker than at Earth. Although at the orbit of Saturn the energy density of the IMF had decreased by two orders of magnitude over its value at $1 \mathrm{AU}$, the solar wind magnetic and electric fields are significant for the kronian magnetosphere.

Most of the existing models of Saturn's magnetosphere (e.g. Connerney et al., 1981a, b, 1983; Behannon et al., 1983; Maurice and Engel, 1995; Bunce and Cowley, 2003) include a ring current in the equatorial plane which is axisymmetric in a first approximation. The flux function for the ring current distribution was obtained numerically by Connerney et al. (1983) from a fit to Voyager magnetometer data. Connerney et al. (1982) used the model of Saturn's internal field characterized by the Schmidtnormalized spherical harmonic coefficients $g_{1}^{0}=21,535 \mathrm{nT}$, $g_{2}^{0}=1,642 \mathrm{nT}, g_{3}^{0}=2,743 \mathrm{nT}$, which are extremely efficient in representing the main magnetic field of Saturn and reconciling the in-situ magnetic field observations obtained by Pioneer 11 , Voyager 1 and 2 . In the model $Z_{3}$ by Connerney et al. (1983), based on Voyager data, the axisymmetric interior source dipole, quadrupole, and octupole terms with a slightly tilted, rotating, nonaxisymmetric uniform field for the exterior sources were used. Davis and Smith (1986) have explored various axisymmetric models of the Pioneer 11 data and also models containing a few nonaxisymmetric terms. A better model of Saturn's magnetic field inside $8 R_{S}$, the SPV model, has been derived by combining the data from Pioneer 11, and Voyager 1 and 2 encounters (Davis and Smith, 1990). The parts of the model field due to external sources are approximated by fields that are uniform but are not parallel to Saturn's rotation axis. Maurice and Engel (1995) model includes three magnetic fields: a dipole field, a ring current field deduced from Voyager observations, and a field of the magnetopause currents.

However, even the first Pioneer 11 flyby provided strong evidence for an open magnetotail configuration (McDonald et al., 1980). Behannon et al. (1981) constructed a semi-quantitative model of Saturn's magnetosphere consistent with the Voyager 1 magnetic field observations and closure of field lines across the tail. Bunce et al. (2003) estimated the magnetopause and tail current system empirically by scaling a model of the Earth's magnetosphere to Saturn. It was shown that modification and scaling of terrestrial magnetic field models may represent a useful approach to modeling the three-dimensional magnetic field at Saturn.

The goal of this paper is to describe a method of calculation of the paraboloid Saturnian magnetospheric model parameters for the chosen epoch. This model includes the internal planet's magnetic field, the ring current field, the field of the tail current system, and of the magnetopause currents shielding all magnetospheric field sources. The model takes into account the interaction with the solar wind, and is developed on the basis of the terrestrial (Alexeev, 1978, 1986) and Jovian (Belenkaya, 2003, 2004; Alexeev and Belenkaya, 2005) magnetospheric models. In particular, this is the first kronian magnetospheric model which includes not only magnetopause and ring currents, but also a tail current system. On the example of the Pioneer 11 flyby data, we show, how the model input parameters could be determined. The magnetospheric magnetic field calculated by using the obtained parameters is compared with the inbound Pioneer 11 data.

\section{Model}

The frequently used empirical model developed for the Earth by Tsyganenko (Tsyganenko, 1995, 1996) is constructed by minimizing the root mean-square (rms) deviation from observations. The large magnetospheric database, which contains the Earth's magnetospheric magnetic field measurements, 
accumulated over many years, was used for this purpose. This method of construction of the "average" magnetospheric model is not appropriate for Saturn, because now we have a small amount of data for successful interpolation, and also due to the strong time dependence of the external magnetospheric magnetic field sources in the kronian magnetosphere.

This is why we used another method of the Saturnian model construction. This method is based on a priori information about the global magnetospheric current systems. Each current system is included as a separate block (module) in the magnetospheric model. As it was shown by the spacecraft flights to Saturn, there are three current systems which are the main contributors to the external kronian magnetospheric magnetic field: magnetopause currents, ring current, and tail current sheet. The paraboloid model initially developed for the Earth (Alexeev, 1978, 1986) was based on an analytical solution of the Laplace equation for each of the large-scale current systems in the magnetosphere with a fixed shape (paraboloid of revolution). The paraboloid model input parameters characterize these magnetospheric current systems (their intensities and locations). Input parameters are determined from empirical data: the solar wind and polar cap observations. The paraboloid magnetospheric model uses a small number of input parameters (less than 10). Such an approach allows us to use the paraboloid model for any variable conditions in the solar wind and inside the magnetosphere, so it can be applied for the terrestrial magnetosphere (Alexeev, 1978; Alexeev et al., 1996, 2001), as well as for the Jovian (Belenkaya, 2003, 2004; Alexeev and Belenkaya, 2005) and kronian ones (taking into account their peculiarities).

The terrestrial paraboloid model was successfully applied, for example, for a description of magnetic storms (Alexeev et al., 1996), for the calculation of locations of simultaneous polar aurorae in both hemispheres for northward IMF (Cumnok et al., 2005), for the explanation of the magnetospheric responses to coronal mass ejection encounters under specific conditions in the interplanetary magnetic field (Clauer et al., 2001; Belenkaya et al., 2004), for the simulation of thetaaurora (Blomberg et al., 2005), etc.

For Jupiter, a global paraboloid model of the magnetospheric magnetic field and a structure of the large-scale plasma flows were constructed for different IMF orientations. The paraboloid model showed that for southward IMF, the anti-sunward flows exist at the flanks and in the distant tail. These flows were summarized with corotation and created the anti-corotation (supercorotation) motion in the morning (dusk) sector of the magnetospheric equatorial plane. In the frame of the paraboloid model, the anticorotation flows in the morning sector of the equatorial magnetosphere, and the anti-corotation and anti-sunward flows in the prenoon polar caps observed by Ulysses were explained by reconnection with southward IMF. The observation of such flows during the Ulysses flyby and their absence dur- ing the Voyager flybys may be caused by different orientations of IMF for these events. The Voyager observations of the corotation flow throughout most of the Jovian magnetodisk plasma sheet, from the subsolar magnetopause to $\sim 150 R_{\mathrm{J}}$, on the nightside and the nightside outflow beyond $\sim 150 R_{\mathrm{J}}$ were easily explained by the reconnection with the northward solar wind magnetic field ( $R_{\mathrm{J}}$ is Jupiter's radius). The electric field distributions caused by the Jovian rotation and the solar wind MHD-generator were constructed (Belenkaya, 2003, 2004). The model calculations yielded the location of the main auroral oval (at co-latitude of $\sim 16^{\circ}$ ), which is caused by the outward field-aligned currents connected with the maximum of the field-aligned potential electric drops (Belenkaya, 2003, 2004; Alexeev and Belenkaya, 2005).

The paraboloid model of Saturn was applied for the description of the Cassini SOI orbit (Alexeev et al., 2006). It also allowed one to analyze the kronian polar cap dynamics influenced by the solar wind and its magnetic field (Belenkaya, 2006; Belenkaya et al., 2006). Here we will consider the paraboloid Saturnian model in detail.

Galopeau and Lecacheux (2000), based on Pioneer 11 and Voyager 1 and 2 observations, described Saturn's magnetopause by a hyperbola, characterized by a conic focus at $x=5 R_{S}$, a semilatus rectum $L=30.8 R_{S}$ and eccentricity $\epsilon=1.09$. The nose of the magnetopause was located at $19.7 R_{S}$.

Maurice et al. (1996), based on the work of Maurice and Engle (1995), presented an idealized 3-D model of Saturn's magnetopause. The magnetopause shape was applied to Pioneer 11 and Voyagers observations. The parametrization of Saturn's magnetopause was extended to nonzero dipole tilt angles. In the models of Maurice and Engle (1995) the ratio of the distance from the magnetopause to the planet in the dawn-dusk meridian to the subsolar distance is 1.3 for Earth and Saturn. Maurice and Engle (1995) showed that Saturn's magnetopause is closer to the Earth's magnetopause than to Jupiter's magnetopause.

An average bow shock to magnetopause stagnation point distance ratio is 1.29 for Saturn, 1.33 for Earth, and $1.22-1.26$ for Jupiter's magnetosphere; it has been suggested that this ratio provides a semi-quantitative measure of the "degree of bluntness" of the front-side magnetosphere (Behannon et al., 1983). Thus, Saturn's magnetosphere, from this point of view, should be less blunt than the Earth's but more blunt than Jupiter's. According to the Pioneer 11 data, Smith et al. (1980) noted that Saturn's magnetopause is consistent with a shape which is similar to that of the Earth. As it can be concluded from the results obtained by Hendricks et al. (2005), the paraboloid of revolution gives a good approximation for the shape of the magnetopause (with accuracy of $\sim 10 \%$ ).

We use, as in the case of the Earth (Alexeev, 1978, 1986) and Jupiter (Belenkaya, 2003, 2004; Alexeev and Belenkaya, 2005), a paraboloid of revolution 
to describe the magnetopause of Saturn. The equation of the paraboloid approximating the magnetopause is: $x / R_{\mathrm{SS}}=1-\left(y^{2}+z^{2}\right) / 2 R_{s s}^{2}$. Here we use the solarmagnetospheric coordinates with the $X$-axis directed to the Sun, Saturn's magnetic moment $\mathbf{M}_{S}$ in the $X Z$ plane, and $Y$ is in the direction of $[\mathbf{Z} \times \mathbf{X}] ; R_{s S}$ is a parameter characterizing the magnetospheric scale (the distance to Saturn's subsolar point). As it was pointed by Behannon et al. (1983), the additional analysis and observations are required to predict with confidence the three-dimensional shape of the dayside magnetopause of Saturn.

The main contributors to the model magnetospheric magnetic field are the following:

1 Saturn's intrinsic magnetic field (dipole), as well as the shielding magnetopause current, which confines the dipole field inside the magnetosphere;

2 The tail currents and their closure currents on the magnetopause;

3 The ring current and the corresponding shielding magnetopause current;

4 The IMF penetrating into the magnetosphere.

The following equations for the magnetic field and electric current density, $\operatorname{div} \mathbf{B}=0$ and $\operatorname{div} \mathbf{j}=0$, are true for all model calculations.

The magnetic field vector $\mathbf{B}_{m}$ is calculated by summing the fields of magnetospheric origin and the penetrated portion of the IMF:

$$
\begin{aligned}
\mathbf{B}_{m}= & \mathbf{B}_{d}\left(B_{S 0}, R_{S}, \Psi\right)+\mathbf{B}_{s d}\left(B_{S 0}, R_{S}, R_{s s}, \Psi\right) \\
& +\mathbf{B}_{T S}\left(R_{s s}, R_{t}, B_{t}, \Psi\right)+\mathbf{B}_{r c}\left(B_{l o}, l_{o}, l_{i}, \Psi\right) \\
& +\mathbf{B}_{s r c}\left(R_{s s}, B_{l o}, l_{o}, l_{i}, \Psi\right)+\mathbf{b}\left(k_{S}, \mathbf{B}_{I M F}\right) .
\end{aligned}
$$

Here $\mathbf{B}_{d}\left(B_{S 0}, R_{S}, \Psi\right)$ describes a pure tilted dipole field; $\mathbf{B}_{s d}\left(B_{S 0}, R_{S}, R_{S S}, \Psi\right)$ is the field of current on the magnetopause shielding the dipole field; $\mathbf{B}_{T S}\left(R_{s s}, R_{t}, B_{t}, \Psi\right)$ is the field of the magnetospheric tail current system (cross-tail currents and their closure magnetopause currents); $\quad \mathbf{B}_{r c}\left(B_{l o}, l_{o}, l_{i}, \Psi\right)$ is a field of Saturn's ring current placed in the equatorial magnetospheric plane; $\mathbf{B}_{s r c}\left(R_{s s}, B_{l o}, l_{o}, l_{i}, \Psi\right)$ is the field of the current on the magnetopause shielding the ring current field; $\mathbf{b}\left(k_{S}, \mathbf{B}_{I M F}\right)$ is a part of the interplanetary magnetic field $\mathbf{B}_{I M F}$ penetrating into the magnetosphere with a coefficient of penetration $k_{S}$.

The Saturn's magnetospheric magnetic field (Eq. 1) is defined by the time-dependent input parameters: the distance from the Saturn center to the subsolar point on the magnetopause, $R_{S S}$; the distances to the outer and inner edges of the ring current, $l_{o}$ and $l_{i}$, respectively; the dipole tilt angle $\Psi$ (the angle between magnetic dipole and axis $Z$ of the solar-magnetospheric coordinate system); the distance from the planet's center to the inner edge of the magnetospheric tail current sheet, $R_{t}$; the magnitude of the field of the tail currents at the inner edge of the tail current sheet, $B_{t} / \alpha_{0}$, where $\alpha_{0}=\left(1+2 R_{t} / R_{s S}\right)^{1 / 2}$; the radial component of the ring current magnetic field at the outer edge of the ring current, $B_{l o}$; the interplanetary magnetic field vector, $\mathbf{B}_{I M F}$, and the coefficient of its penetration into the magnetosphere, $k_{S}$. Away from each thin current sheet, the magnetic field of the corresponding magnetospheric source (magnetopause, ring and tail currents) may be represented in terms of the gradient of a scalar magnetic potential.

\section{Analytical approximate description of different mag- netospheric magnetic field sources along the Saturn- Sun line}

The input model parameters are different for different spacecraft flybys. They change even along a single orbit. For example, the average stand-off distance of the magnetopause at Saturn, determined by the Pioneer 11 and Voyagers data, is $R_{s s}=21 R_{S}$ (Behannon et al., 1983), i.e. at the orbit of Titan (Acuña et al., 1980). As the position of the subsolar magnetopause varies considerably, depending on external solar wind and internal kronian magnetospheric conditions, Titan is not always inside the magnetosphere.

All paraboloid model parameters have a simple physical sense and can be determined from observations (directly or indirectly). If determination of some physical values coinciding with the input parameters of Saturn's paraboloid model, have been done earlier by other authors, we use these results to give the corresponding references.

Here we determine the model parameters for the Pioneer 11 passage. While the internal planet's field is comparatively stable, the input model parameters for the external sources of the kronian magnetospheric magnetic field are influenced by the solar wind and internal magnetospheric conditions. Giampieri and Dougherty (2004) showed that it is necessary to use different sets of parameters for the inbound and outbound sections of any flyby (Pioneer 11, Voyager 1 and 2). Here we study the inbound section of the Pioneer 11 trajectory, which was located slightly above the equatorial plane near a local noon (the outbound portion was extended along the dawn meridian).

\subsection{Planet's magnetic field}

In Fig. 1 a solid black curve represents the magnetic field module measured by the Pioneer 11 Helium Vector Magnetometer (HVM) during the inbound pass obtained from NASA's Planetary Data System (http://pds.jpl.nasa.gov/). The magnetopause position and the point of closest approach to Saturn are marked by the vertical dashed lines with labels "MP" and "CA", respectively. Along the horizontal axis the day number of the year (DOY), and corresponding distance from the planet in Saturn's radii $\left(R_{S}\right)$, are noted. We see that the magnetic field at the subsolar magnetopause is very 
variable. We determine the average value of the field at the noon magnetopause, $<\left.B^{o b s}\right|_{R_{s s}}>$, as an arithmetic mean between the closest field peaks on the both sides of the magnetopause. The corresponding values are $7.4 \mathrm{nT}$ at $r=17.84 R_{S}$ out of the magnetopause, and $11.3 \mathrm{nT}$ at $r=17.15 R_{S}$ inside the magnetopause. Thus, $<\left.B^{o b s}\right|_{R_{s s}}>=((11.3+7.4) / 2) \mathrm{nT}$ $=9.35 \mathrm{nT}$ can be considered as a character magnetospheric magnetic field value at the subsolar magnetopause.

Following Sauer et al. (2004), here we assume that a dipole magnetic field (with equatorial magnetic field $B_{S 0}=21160 \mathrm{nT}$ ) represents a good approximation in the regions located out of the immediate proximity of the planet's surface. Thus, Saturn's dipole magnetic field at the $X$ axis could be written as:

$\mathbf{B}_{d}=-B_{S 0}\left(R_{S} / r\right)^{3}$.

In our analytic calculations we consider $\Psi=0$ (the correct value of $\Psi=0.95^{\circ}$ for the Pioneer 11 epoch is taken into account in the paraboloid model precise computation, see Sect. 6). The unperturbed dipole field at Saturn's subsolar magnetopause is

$\left.B_{d}\right|_{R_{s s}}=-B_{S 0}\left(R_{S} / R_{s S}\right)^{3}$.

We see that $\left.B_{d}\right|_{R_{s s}}$ decreases with the growth of the input model parameter $R_{s s}$, and consequently, with the decrease in the solar wind dynamic pressure. From the Pioneer 11 data (see Fig. 1) we determine $R_{s s} \sim 17.5 R_{S}$. The corresponding dipole field at the subsolar point is $-3.95 \mathrm{nT}$ (see Eq. 3).

3.2 Magnetic field of the magnetopause current screening the dipole field

For the estimation of the magnetic field of the ChapmanFerraro current screening the dipole field, by analogy with the terrestrial magnetospheric model (see, for example, formula (8) in Clauer et al., 2001), we can write an approximate formula for Saturn:

$$
\begin{aligned}
B_{s d-z} & =-0.7 B_{S 0}\left(R_{S} / R_{s s}\right)^{3}\left(1+x / R_{s s}\right) \\
& =-0.7\left(M_{S} / R_{s s}^{3}\right)\left(1+x / R_{s s}\right) .
\end{aligned}
$$

Here, $B_{s d-z}$ is the $z$ component of the screening current field calculated at the Saturn-Sun line $(X) ; M_{S}=B_{S 0} R_{S}^{3}$ is the dipole magnetic moment of Saturn. For example, the contribution of the Chapman-Ferraro current to the magnetic field at the subsolar point for the Pioneer 11 inbound pass is

$\left.B_{s d-z}\right|_{R_{s s}}=-1.4 B_{S 0}\left(R_{S} / R_{s s}\right)^{3}=-5.53 \mathrm{nT}$.

Here for rough estimations we consider the dipole to be located on Saturn's axis of rotation and directed northward normal to the ring plane. It could be mentioned that $\left.B_{s d-z}\right|_{R_{s s}}$ also decreases with the growth of the input model parameter $R_{s s}$, and consequently, with the decrease in the solar wind dynamic pressure.

\subsection{Tail current system magnetic field}

Saturn's magnetosphere, in a first approximation, is azimuthally symmetric inside $L=15$ (Smith et al., 1980; Ness et al., 1981), where $L$ is an equatorial distance from the planet's center measured in planet radii. Asymmetries, due to the magnetopause and tail current systems, driven by the solar wind interaction, become evident in the Voyager magnetic field observations at radial distances greater than $\sim 15 R_{S}$ (outbound) (Connerney et al., 1983). In spite of the fact that there are no direct observations of the tail current sheet separated kronian tail lobes, the spacecraft data show the existence of a magnetotail of at least $80 R_{S}$ in diameter at the time of the Voyager 1 encounter, expanding to $\sim 140 R_{S}$ or more during the Voyager 2 encounter (Behannon et al., 1983). The estimated length of Saturn's magnetic tail is $\sim 1500 R_{S}$ (Cowley et al., 2004).

Here we use the Pioneer 11 flyby data to calculate the parameter values for the tail current system. In the data of the inbound portion of the Pioneer 11 trajectory there was no evidence for a thin equatorial current sheet (only a dipole under compression by the solar wind), however, the outbound observations showed the existence of such sheet. A last closed field line at $L=20$ corresponds to an invariant latitude of $\cos ^{-1}(1 / L)=77^{\circ}$ (Smith et al., 1980). (Using the nightside outbound Voyager 1 data, Ness et al. (1981) derived an invariant latitude of $75-79^{\circ}$ for the open-closed field line boundary.) If the magnetic flux above this latitude, $F_{p c}$, is responsible for a magnetic tail, we can calculate the model parameter $B_{t}$.

For the calculation we use a formula: $B_{t}=2 F_{\infty} /\left(\pi R_{s s}^{2} \alpha_{0}\right)$ (Alexeev et al., 2003), where $F_{\infty}$ is a magnetic flux in the magnetospheric tail lobe; $\alpha_{0}=\left(1+2 R_{t} / R_{s s}\right)^{1 / 2}$. Assuming $F_{\infty}=F_{p c}=2 B_{S 0} \pi R_{S}^{2} \sin ^{2} \theta_{m}$, where $2 B_{S 0}$ is the magnetic field in Saturn's polar regions caused by the dipole, $\theta_{m}$ is a co-latitude of the ionospheric boundary between the open and closed field lines, and $\pi R_{S}^{2} \sin ^{2} \theta_{m}$ is the polar cap area, we obtain:

$B_{t}=\frac{4 B_{S 0} \sin ^{2} \theta_{m}}{\sqrt{1+2 R_{t} / R_{s s}}}\left(\frac{R_{S}}{R_{s s}}\right)^{2}$

$\left(B_{S 0}=21160 \mathrm{nT}, R_{S}=60330 \mathrm{~km}\right)$.

Analyzing the outbound Pioneer 11 data (Smith et al., 1980 ), we can find that beyond $\sim-14 R_{S}$, the magnetic field configuration is similar to that which is attributed to the presence of an equatorial current sheet. Thus, for the Pioneer 11 flyby, we choose the value of parameter $R_{t}$ to be equal to $14 R_{S}$, and for $R_{\mathrm{ss}}=17.5 R_{S}$, and $\theta_{m}=13^{\circ}$, we obtain $\alpha_{0}=1.61$ and $B_{t}=8.69 \mathrm{nT}$.

For the terrestrial magnetosphere, Alexeev et al. (2000) presented an approximate formula for the dependence of the tail current sheet magnetic field at the Earth-Sun line on the 


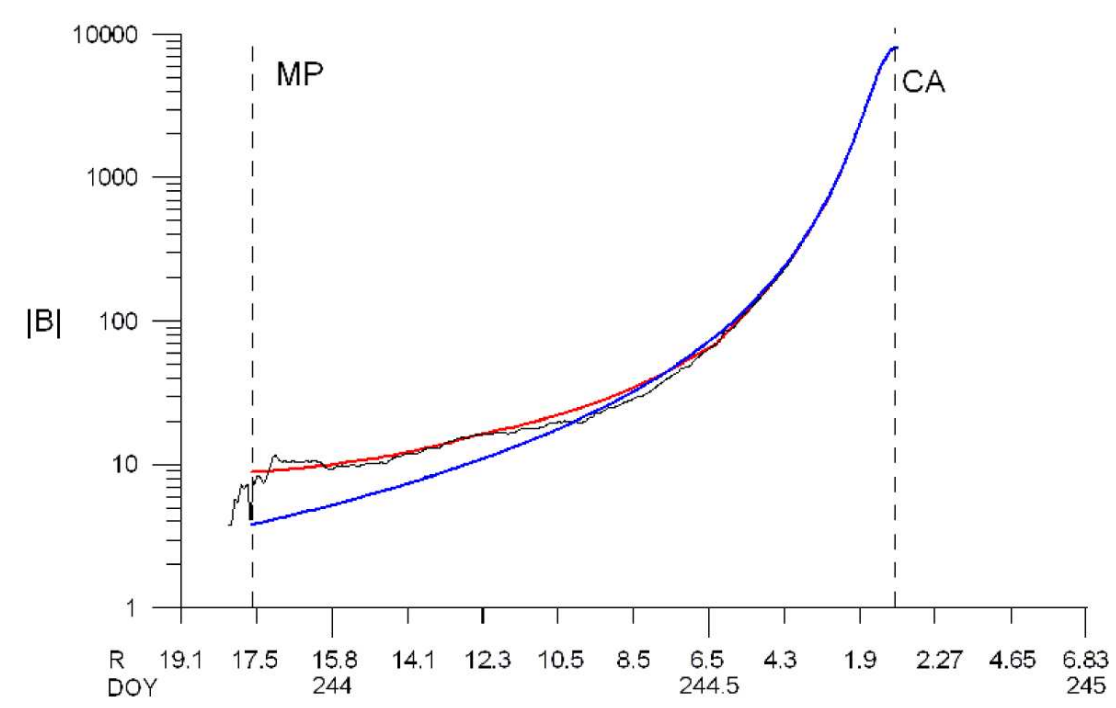

Fig. 1. Magnetic field module measured by Pioneer 11 Helium Vector Magnetometer (HVM) during the inbound pass (black curve); magnetic field module calculated by the SPV model (blue curve), and computed by the kronian paraboloid model with parameters selected by using the approximate formulas (red curve). Data of Helium Vector Magnetometer measurements during the Pioneer 11 mission were obtained from the NASA's Planetary Data System (http://pds.jpl.nasa.gov/). Vertical dashed lines with lables "MP" and "CA" mark the magnetopause and the closest approach, respectively. Along the horisontal axis the day number of the year (DOY), and corresponding distance from the planet in the Saturn's radii $\left(R_{S}\right)$ are noted. Along the vertical axis the module of the magnetic field, measured in logarithmic scale is given. The input model parameters for the inbound Pioneer 11 pass: $R_{S S}=17.5 R_{S} ; l_{o}=12.5 R_{S} ; l_{i}=6.5 R_{S} ; \Psi=0.8^{\circ} ; R_{t}=14 R_{S} ; B_{t}=8.7 \mathrm{nT}$; $B_{l o}=3.62 \mathrm{nT}$.

geocentric distance $x$. Here we use an analogy approximate formula rewritten for the kronian magnetosphere:

$B_{T S}=\frac{B_{t}}{\alpha_{0}}\left\{\begin{array}{l}\exp \left\{-\frac{x+R_{t}}{R_{s s}}\right\} \text { for } x>-R_{t}, \\ \exp \left\{2 \frac{x+R_{t}}{R_{s s}}\right\} \text { for } x<-R_{t},\end{array}\right.$

where the tail current system magnetic field is presented at the Saturn-Sun line, dependent on the distance $x$ from Saturn's center. In particular, the contribution of the tail current system to the magnetic field at the subsolar point for the Pioneer 11 inbound flyby is

$\left.B_{T S}\right|_{R_{s s}}=\frac{B_{t}}{\alpha_{0}} \exp \left\{-\frac{R_{s s}+R_{t}}{R_{s s}}\right\}=0.89 \mathrm{nT}$

for the chosen model parameters: $R_{s s}=17.5 R_{S}, R_{t}=14 R_{S}$, $B_{t}=8.69 \mathrm{nT}\left(\alpha_{0}=1.61\right)$. We see that the contribution of the tail current system to the subsolar magnetic field is proportional to the input model parameter $B_{t}$, which, according to Eq. (6), increases with an increase in the tail lobe flux and a decrease in the distance to the subsolar point $R_{s s}$ (increase in the solar wind pressure).

\subsection{Magnetic field of the ring current}

Closer to Saturn $\left(r<10 R_{S}\right)$ the observed field by Pioneer 11 was less than the dipole field, which might be evidence for a distributed ring current encircling Saturn (Smith et al., 1980). A similar depression in the magnetospheric field strength was observed by both Voyagers (Ness et al., 1981).

According to the Bunce and Cowley (2003) estimations, for the Pioneer 11 epoch, the ring current was located between 6.5 and $12.5 R_{S}$, had a north-south extent of $4 R_{S}$, and carried a total current of 9.6 MA. According to the Giampieri and Dougherty (2004) model, the inner and outer radii of the ring current for Pioneer 11 were $6.4 R_{S}$ and $13.9 R_{S}$, respectively, and the ring current thickness was $3.6 R_{S}$. For the inbound section of the Pioneer 11 trajectory Giampieri and Dougherty (2004) gave the values of $6.8 R_{S}$ and $12.2 R_{S}$ for the inner and outer radii of the ring current, respectively.

For comparison we can note that the ring current inferred from the Voyager 1 data had inner and outer boundaries of 8.5 and $15.5 R_{S}$ in the equatorial plane, with a vertical thickness $D$ of $5 R_{S}$ (Sittler et al., 1983). In order to better fit the Voyager 2 observations, the inner edge of the ring current has been decreased to $8 R_{S}$ and the thickness $D$ increased to $6 R_{S}$. The total azimuthal current was 11.5 MA (Connerney et al., 1983).

No Saturn satellite plays a role equivalent to Io for the Jovian magnetosphere. The situation in the kronian magnetosphere is complicated by the presence of multiple plasma sources rather than a single dominant source as at Jupiter. Possible sources of Saturnian thermal plasma are: the rings, the inner icy moons, Titan's and Saturn's atmospheres. 
Richardson (1986) suggested that a major plasma source is at $L \approx 10$ and that sporadic rapid transport takes place outside this source. The rate of magnetospheric plasma generation at Saturn, estimated by Hill (2001), is $\sim 300 \mathrm{~kg} / \mathrm{s}$, and by Sauer et al. (2004) for $L>12$ is $\sim 1.6 \mathrm{~kg} / \mathrm{s}$ and $\sim 6.3 \mathrm{~kg} / \mathrm{s}$, according to the Voyager 1 and Voyager 2 data, respectively (for Jupiter it is $\sim 1 \mathrm{~T} / \mathrm{s}$ ).

Here we use the scaled replica of Jupiter's magnetodisk model (Belenkaya, 2003, 2004; Alexeev and Belenkaya, 2005). Rapid planetary rotation coupled with centrifugallydriven mass outflow from the inner magnetosphere leads to the creation of the kronian ring current. We assume that a very thin ring current is centered at Saturn's center and is symmetrical about the kronian dipole axis. The azimuthal current between $l_{i}$ and $l_{o}$ varies as the inverse square of the radial distance, $r$. By analogy with Eq. (26) in Belenkaya et al. (2005) for Jupiter, we can determine the azimuthal current per unit radial distance in the kronian ring current as

$i_{\varphi}=\frac{2 B_{l o}}{\mu_{0}}\left(\frac{l_{o}}{r}\right)^{2}$

where a spherical polar coordinate system $(r, \theta, \varphi)$ is used $(\theta$ is a polar angle measured from the axis of the ring current, and $\varphi$ is in the sense of the planet's rotation). Integration over this current gives an effective magnetic moment of the Saturnian ring current, $\mu_{r c}$ :

$\mu_{r c}=\pi \int_{l_{i}}^{l_{o}} i_{\varphi} r^{2} d r=\frac{4 \pi}{\mu_{0}} \frac{B_{l o}}{2} l_{o}^{3}\left(1-\frac{l_{i}}{l_{o}}\right)$.

If we introduce the notations $B_{r c 0}$ and $M_{r c}$ :

$B_{r c 0}=\frac{B_{l o}}{2}\left(1-\frac{l_{i}}{l_{o}}\right)$,

and

$M_{r c}=\mu_{0} \mu_{r c} / 4 \pi=B_{r c 0} l_{o}^{3}$,

then we can roughly determine the magnetic field of the ring current by the equations

$B_{r c-z}=-M_{r c} / r^{3}=-B_{r c 0} l_{o}^{3} / r^{3}$ for $r \geq l_{o}$.

and

$B_{r c-z}=2 M_{r c} / l_{i}^{3}=2 B_{r c 0} l_{o}^{3} / l_{i}^{3}$ for $r \leq l_{i}$.

The total current, $J_{\varphi}$, in the Saturnian ring current could be obtained from the integration:

$J_{\varphi}=\int_{l_{i}}^{l_{o}} i_{\varphi} d r=\frac{2}{\mu_{0}} B_{l o} \frac{l_{o}^{2}}{l_{i}}\left(1-\frac{l_{i}}{l_{o}}\right)$.

From Eqs. (10) and (15) we find a relation between the effective magnetic moment of the ring current, $\mu_{r c}$, and the total current in it, $J_{\varphi}$, for the distribution of the azimuthal current, $i_{\varphi}$, given by Eq. (9):

$\mu_{r c}=\pi J_{\varphi} l_{o} l_{i}$.
Connerney et al. (1981b, 1983) scaled their model of the Jovian magnetodisk (Connerney et al., 1981a) to use it for the Saturnian ring current. Connerney et al. (1981b, 1983) applied a $1 / r$ dependence for the current density. Their model was obtained from the Voyager 1 and 2 observations. The ring current was located between 8 and $15.5 R_{S}$ and its density was written as:

$j_{\varphi}=I_{0} / r$

where a current strength was $I_{0}=2.9 \cdot 10^{6} \mathrm{~A} / R_{S}$. These model parameters were selected to best fit the Voyagers data set. The total integrated ring current was $11.5 \cdot 10^{6} \mathrm{~A}$.

If we also took the inverse radial distance dependence of the azimuthal current per unit radial distance in the kronian ring current $i_{\varphi}^{\prime}$, then from the equation: $\operatorname{curl} \mathbf{B}^{\prime}=\mu_{0} \mathbf{i}^{\prime}$, we obtain at the outer edge of the ring current: $2 B_{l o}^{\prime}=\left.\mu_{0} i_{\varphi}^{\prime}\right|_{l_{o}}$, and consequently

$i_{\varphi}^{\prime}=\frac{2 B_{l o}^{\prime}}{\mu_{0}}\left(\frac{l_{o}}{r}\right)$.

The corresponding expressions for an effective ring current moment, $\mu_{r c}^{\prime}$, and the total current, $J_{\varphi}^{\prime}$, are

$\mu_{r c}^{\prime}=\pi \int_{l_{i}}^{l_{o}} i_{\varphi}^{\prime} r^{2} d r=\frac{\pi}{\mu_{0}} B_{l o}^{\prime} l_{o}^{3}\left(1-\left(l_{i} / l_{o}\right)^{2}\right)$

and

$J_{\varphi}^{\prime}=\int_{l_{i}}^{l_{o}} i_{\varphi}^{\prime} d r=\frac{2 B_{l o}^{\prime} l_{o}}{\mu_{0}} \ln \frac{l_{o}}{l_{i}}$.

From Eq. (19) we find

$M_{r c}=\mu_{0} \mu_{r c}^{\prime} / 4 \pi=\frac{B_{l o}^{\prime}}{4} l_{o}^{3}\left(1-\left(l_{i} / l_{o}\right)^{2}\right)=B_{r c 0} l_{o}^{3}$,

where,

$B_{r c 0}=\frac{B_{l o}^{\prime}}{4}\left(1-\left(l_{i} / l_{o}\right)^{2}\right)$.

Later we will estimate the difference in the ring current parameter values due to these different dependences of the ring current densities, $i_{\varphi}$ and $i_{\varphi}^{\prime}$, on the radial distance, $r$ (Eqs. 9, $18)$.

3.5 Magnetic field of the magnetopause current screening the ring current field

By analogy with Eq. (4) in zero approximation we can estimate along the Saturn-Sun line the $z$-component of magnetic field $B_{s r c}$ of the magnetopause currents shielding the ring current field:

$B_{s r c-z}=-0.7\left(M_{r c} / R_{s s}^{3}\right)\left(1+\frac{x}{R_{s s}}\right)$,

where $M_{r c}$ is determined by Eqs. (10) and (12). 


\section{Calculations by approximate formulas for the Pio- neer 11 inbound flyby}

Using the approximate formulas of the magnetospheric magnetic fields of different sources along the Saturn-Sun line (Eqs. 2, 4, 7, 13, and 23), we can obtain the unknown parameter $B_{l o}$ from the comparison of the calculated total magnetospheric magnetic field at the subsolar point (as a function of $B_{l o}$ ) with the observations. Saturn's dipole, the magnetopause current screening it, and the tail current system fields at this point are given by Eqs. (3), (5), and (8), respectively. The ring current and its screening current magnetic fields at the subsolar magnetopause can be presented as:

$\left.B_{r c}\right|_{R_{s s}}=-\left(M_{r c} / R_{s s}^{3}\right)$

and

$\left.B_{s r c-z}\right|_{s s}=-1.4 B_{l o}\left(l_{o} / R_{s s}\right)^{3}$,

where $B_{r c 0}$ is determined by Eq. (11), and $M_{r c}$ by Eq. (12). The magnetic field of both these sources at the subsolar point is

$\left.B_{r c}\right|_{R_{s s}}+\left.B_{s r c-z}\right|_{R_{s s}}=-2.4 B_{r c 0}\left(l_{o} / R_{s s}\right)^{3}$.

Under typical conditions the interplanetary field in the kronian magnetosheath is principally azimuthal. During the Pioneer 11 inbound path, the IMF was draped around the magnetosphere with a latitudinal angle near zero and a longitudinal angle approximately $270^{\circ}$ (Smith et al., 1980). Thus, we can assume $b_{z} \sim 0$ for this case, and the total magnetic field at the subsolar point is

$\left.B_{z}\right|_{R_{s s}}=-2.4 B_{S 0}\left(R_{S} / R_{s s}\right)^{3}$

$+\frac{B_{t}}{\alpha_{0}} \exp \left\{-\frac{R_{s s}+R_{t}}{R_{s s}}\right\}-2.4 B_{r c 0}\left(l_{o} / R_{s s}\right)^{3}$.

As mentioned earlier, we chose $<\left.B^{o b s}\right|_{R_{s s}}>=9.35 \mathrm{nT}$ as a character value describing the observed magnetic field at the subsolar kronian magnetopause. Using Eqs. (3), (5), and (8), and taking $\left.B_{z}\right|_{R_{s s}}=<\left.B^{o b s}\right|_{R_{s s}}>$, we can present Eq. (27) as

$$
-9.35 \mathrm{nT}=-3.95 \mathrm{nT}-5.53 \mathrm{nT}+0.89 \mathrm{nT}-2.4 B_{r c 0}\left(l_{o} / R_{s s}\right)^{3} \text {, }
$$

or $0.76 \mathrm{nT}=2.4 B_{r c 0}\left(l_{o} / R_{S S}\right)^{3}$. For the chosen parameter values: $l_{o}=12.5 R_{S}$ and $R_{S s}=17.5 R_{S}$, we obtain $B_{r c 0}=0.87 \mathrm{nT}$, and from Eq. (11) for $l_{i}=6.5 R_{S}$ we derive $B_{l o}=3.62 \mathrm{nT}$. The corresponding value of the effective magnetic moment of the ring current (Eq. 12) is $M_{r c}=1699,22 \mathrm{nT} \cdot R_{S}^{3}$, and

$M_{r c} / M_{S}=1699,22 \cdot R_{S}^{3} / 21160 \cdot R_{S}^{3}=0.08$.

So, for Saturn at the Pioneer 11 epoch, our estimation of the ratio of the ring current effective magnetic moment, $M_{r c}$, to the moment of the planet, $M_{S}$, gives $\sim 0.1$ (according to Bunce and Cowley (2003), an effective magnetic moment of the kronian ring current is $\sim 0.21 M_{S}$ ). For Jupiter this ratio is of the order of 2.6 (Belenkaya, 2003, 2004; Alexeev and Belenkaya, 2005).

Thus, all paraboloid model parameter values for the Pioneer 11 inbound flyby are determined: the distance from Saturn's center to the subsolar point on the magnetopause, $R_{S S}=17.5 R_{S}$; the distances to the outer and inner edges of the ring current, $l_{o}=12.5 R_{S}$ and $l_{i}=6.5 R_{S}$, respectively; the distance from the planet's center to the inner edge of the magnetospheric tail current sheet, $R_{t}=14 R_{S}$; the magnitude of the field of the tail current system at the inner edge of the tail current sheet, $B_{t} / \alpha_{0}$, where $\alpha_{0}=1.61$ and $B_{t}=8.69 \mathrm{nT}$; the radial component of the ring current magnetic field at the outer edge of the ring current, $B_{l o}=3.62 \mathrm{nT}$.

\section{Comparison of calculations by approximate formulas with the Pioneer 11 data}

We can compare our calculations by approximate formulas with the observed data during the inbound Pioneer 11 path at selected points. For example, as a first selected point we choose the outer edge of the ring current $\left(r=l_{o}\right)$. The Saturnian dipole field at the outer edge of the ring current (Eq. 2) is $B_{d-z} \mid l_{o}=-B_{S 0}\left(R_{S} / l_{o}\right)^{3}=-10.83 \mathrm{nT}$, and the magnetopause current shielding Saturn's dipole (Eq. 4) is $\left.B_{s d-z}\right|_{l_{o}}=-0.7 B_{S 0}\left(R_{S} / R_{S s}\right)^{3}\left(1+l_{o} / R_{S S}\right)=-4.74 \mathrm{nT}$.

The field caused by the tail current system (Eq. 7) at the outer edge of the ring current is $\left.B_{t-z}\right|_{l_{o}}=\frac{B_{t}}{\alpha_{0}} \exp \left\{-\frac{l_{o}+R_{t}}{R_{S S}}\right\}=1.19 \mathrm{nT}$. The ring current field $z$ component at the outer edge of the ring current is $B_{r c-z} \mid l_{o}=-B_{r c 0}=-0.87 \mathrm{nT}$ (Eq. 13), and the current screening the ring current gives $\left.B_{s r c-z}\right|_{l_{o}}=-0.7 B_{r c 0}\left(l_{o} / R_{S S}\right)^{3}\left(1+l_{o} / R_{S S}\right)=-0.38 \mathrm{nT}$

(see Eq. 23). So, the contribution of all these sources at $r=l_{o}$ is $\left.B_{z}\right|_{l_{o}}=-15.63 \mathrm{nT}$. The value of the magnetic field at this point, measured by the Pioneer 11 , is $\left.B^{o b s}\right|_{l_{o}}=-15.9 \mathrm{nT}$ (the absolute and relative deviations of the calculated values from observations are $\left.\Delta B\right|_{l_{o}}=\left.B_{z}\right|_{l_{o}}-\left.B^{o b s}\right|_{l_{o}}=0.27 \mathrm{nT}$ and $\left.\delta B\right|_{l_{o}}=\left.(\Delta B / B)\right|_{l_{o}} \sim 0.02$, respectively). Thus, we obtain a good coincidence of our result received by approximate formulas with observations.

The second selected point is the inner edge of the kronian ring current, $r=l_{i}$. Magnetic fields of the kronian dipole and its screening current at the inner edge of the ring current are: $\left.\quad B_{d-z}\right|_{i}=-B_{S 0}\left(R_{S} / l_{i}\right)^{3}=-77.05 \mathrm{nT}$, and $\left.B_{s d-z}\right|_{l_{i}}=-0.7 B_{S 0}\left(R_{S} / R_{s s}\right)^{3}\left(1+l_{i} / R_{s s}\right)=-2.76 \mathrm{nT}$, respectively. At the inner edge of the ring current, the field caused by the tail current system (Eq. 7) is $\quad B_{t-z} \mid l_{i}=\frac{B_{t}}{\alpha_{0}} \exp \left\{-\frac{l_{i}+R_{t}}{R_{s s}}\right\}=1.67 \mathrm{nT}$. The magnetic field caused by the ring current at $r \leq l_{i}$ is given by Eq. (14): $\quad B_{r c-z}=2 B_{r c 0} l_{o}^{3} / l_{i}^{3}=12.37 \mathrm{nT}$, and the 
current screening the ring current gives $B_{s r c-z} \mid l_{i}=$ $-0.7 B_{r c 0}\left(l_{o} / R_{s s}\right)^{3}\left(1+l_{i} / R_{s s}\right)=-0.3 \mathrm{nT}$ (see Eq. 23). Contribution of all magnetospheric current systems at $r=l_{i}$ is equal to $B_{z} l_{i}=-66.07 \mathrm{nT}$. The corresponding value, according to the Pioneer 11 inbound data, is $\left.B_{z}^{o b s}\right|_{l_{i}}=-64.13 \mathrm{nT} \quad\left(\left.\Delta B\right|_{l_{i}}=\left.B_{z}\right|_{l_{i}}-\left.B^{o b s}\right|_{l_{i}}=-1.94 \mathrm{nT}\right.$; $\left.\left.\delta B\right|_{l_{i}}=\left.(\Delta B / B)\right|_{l_{i}} \sim 0.03\right)$. Again, we receive good accordance with the observations.

For the Pioneer 11 and Voyagers passes, the presence of a quasi-uniform northward field of $\sim 10 \mathrm{nT}$ (which is a difference between the observed and dipole fields) was registered in the inner region $\left(r<8 R_{S}\right)$ of Saturn's magnetosphere. This northward field was considered as a manifestation of the kronian ring current. Here we can take into account the contributions of all global magnetospheric current systems to this difference between the observed and dipole fields. According to our calculations, this difference at the inner edge of the ring current is $B_{z}\left|l_{i}-B_{d-z}\right| l_{i}=-66.07 \mathrm{nT}+77.05 \mathrm{nT}=10.98 \mathrm{nT}$, which corresponds well to observations ( $B_{z}^{\text {obs }}\left|l_{i}-B_{d-z}\right| l_{i}$ $=-64.13 \mathrm{nT}+77.05 \mathrm{nT}=12.92 \mathrm{nT}$ ). As it was shown above, in reality, the main contribution to this difference gives the ring current $(12.37 \mathrm{nT})$, the magnetopause currents screening the dipole field and the ring current give $-2.76 \mathrm{nT}$, and $-0.30 \mathrm{nT}$, respectively, and the tail current system yields $1.67 \mathrm{nT}$.

Another selected point could be taken, for example, between the subsolar magnetopause and the ring current. We choose $r=15.8 R_{S}$. At $r=15.8 R_{S}$, we receive for the dipole field $B_{d-z} \mid 15.8 R_{S}=-B_{S 0}\left(R_{S} / 15.8 R_{S}\right)^{3}=-5.36 \mathrm{nT}$, and for its screening current field (Eq. 4): $B_{s d-z} \mid 15.8 R_{S}=-0.7 B_{S 0}\left(R_{S} / R_{s s}\right)^{3}\left(1+15.8 R_{S} / R_{S S}\right)$ $=-5.25 \mathrm{nT}$. The tail current system field at this point is $B_{t-z} \mid 15.8 R_{S}=\frac{B_{t}}{\alpha_{0}} \exp \left\{-\frac{15.8 R_{S}+R_{t}}{R_{S s}}\right\}=0.98 \mathrm{nT}$. The ring current field $z$ component is $B_{r c-z} \mid 15.8 R_{S}=-B_{r c 0}$ $\left(l_{o} / 15.8 R_{S}\right)^{3}=-0.43 \mathrm{nT}$ (Eq. 13), and its screening current field is $B_{s r c-z} \mid 15.8 R_{S}=-0.7 B_{r c 0}\left(l_{o} / R_{s S}\right)^{3}\left(1+15.8 R_{S} / R_{s s}\right)$ $=-0.43 \mathrm{nT}$ (see Eq. 23). The total calculated field at $r=15.8 R_{S}$ is $B_{z} \mid 15.8 R_{S} \sim-10.49 \mathrm{nT}$, while the observed field is $\left.B^{\text {obs }}\right|_{15.8 R_{S}}=-10 \mathrm{nT}$ $\left(\left.\Delta B\right|_{15.8 R_{S}}=\left.B_{z}\right|_{15.8 R_{S}}-\left.B^{o b s}\right|_{15.8 R_{S}}=-0.49 \mathrm{nT} ;\left.\delta B\right|_{15.8 R_{S}}\right.$

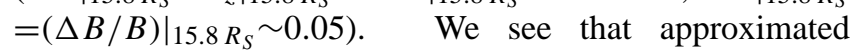
formulas give a rather good result.

From Eq. (15) we can estimate the value of a total current strength in the ring current $J_{\varphi}=\left(2 / \mu_{0}\right) B_{l o}\left(l_{o}^{2} / l_{i}\right)\left(1-\left(l_{i} / l_{o}\right)\right) \sim 4 \mathrm{MA}, \quad$ where $B_{l o}$ $=3.62 \mathrm{nT}$. The corresponding values of $J_{\varphi}^{\prime}$ and $B_{l o}^{\prime}$ for $i_{\varphi}^{\prime}$ proportional to $r^{-1}$ are $J_{\varphi}^{\prime}=\frac{2 B_{l o}^{\prime} l_{o}}{\mu_{0}} \ln \frac{l_{o}}{l_{i}} \sim 6 \mathrm{MA}$ (see Eq. 20), and $B_{l o}^{\prime}=4 B_{r c 0} /\left(1-\left(l_{i} / l_{o}\right)^{2}\right)=4.77 \mathrm{nT}$ (see Eq. 22). Thus, we see that the current density distributions proportional to $r^{-2}$ and $r^{-1}$ do not lead to a very large difference in the ring current parameter values. The total ring current for the Pioneer 11 epoch was 9.6 MA, according to the Bunce and Cowley (2003) result, and the value 6.4 MA could be concluded from Table 1 of Giampiery and Dougherty (2004).

\section{Computation of the magnetospheric magnetic field by the paraboloid kronian model for the Pioneer 11 in- bound flyby}

Precise computation of the magnetic field performed in the kronian paraboloid model along the inbound portion of the Pioneer 11 orbit is demonstrated in Fig. 1 (red curve). The calculation using the SPV model only is shown by the blue curve. The coefficients for the interior planet's source terms for the SPV model are $g_{1}^{0}=21160 \mathrm{nT}, g_{2}^{0}=1560 \mathrm{nT}$, and $g_{3}^{0}=2320 \mathrm{nT}$ for a Saturn radius of $60330 \mathrm{~km}$ (Davis and Smith, 1990). The input model parameters for the inbound Pioneer 11, pass determined by the described method, are: $\quad R_{s s}=17.5 R_{S} ; \quad l_{o}=12.5 R_{S} ; \quad l_{i}=6.5 R_{S} ; \quad \Psi=0.95^{\circ}$; $R_{t}=14 R_{S} ; B_{t}=8.7 \mathrm{nT} ; B_{l o}=3.62 \mathrm{nT}$. We see that the parameters found by using simple approximate formulas imitating the magnetospheric current systems' field, give good accordance with the observations: the rms error is equal to 2.9 for the average value of the magnetic field module $79.3 \mathrm{nT}$, for $2.7 R_{S}<r<17.5 R_{S}$ (the Giampieri and Dougherty (2004) modeled Pioneer 11 magnetic field discarding data within $4 R_{S}$ of Saturn's magnetosphere). By taking into account the effect of the magnetopause and tail currents, this allowed us to obtain good quantitative accordance with the measured data, even in the outer dayside magnetosphere, in distinction from the other models of Saturn's magnetosphere. For example, in the Giampieri and Dougherty (2004) model no other external contributions besides those due to Saturn's disk, have been considered. Giampieri and Dougherty (2004) compared their results for the Pioneer 11 flyby with those obtained by Connerney et al. (1983) and by Bunce and Cowley (2003). In Fig. 3 (upper panel, left side) from the Giampieri and Dougherty (2004) paper it is seen that in the outer Saturnian magnetosphere, the minimum deviation from the observations gives $\sim 4.3 \mathrm{nT}$ in the Bunce and Cowley (2003) model, while the Connerney et al. (1983) model results are absent in this region, and the Giampieri and Dougherty (2004) deviation is $\sim 5.7 \mathrm{nT}$; the paraboloid kronian model gives a deviation of $\sim 2 \mathrm{nT}$ near the dayside magnetopause (see Fig. 1 of the present paper).

Studying Fig. 3 of the Giampieri and Dougherty (2004) paper in the middle magnetosphere, in the region of the kronian ring current, we see that the best fitting is obtained by Connerney et al. (1983) (however, near the inner edge of the ring current the deviation reaches $\sim 8 \mathrm{nT}$ ), while the maximum deviation of Giampieri and Dougherty (2004) is $\sim 4 \mathrm{nT}$ near the outer edge of the ring current; Bunce and Cowley (2003) give $\leq 8 \mathrm{nT}$ in this region, and the paraboloid model's maximum deviation is $\sim 2 \mathrm{nT}$ in the middle of the ring current, and $\sim 7 \mathrm{nT}$ near its inner edge (see Fig. 1 of the present paper). 




Fig. 2. Noon-midnight cross-section of the Saturn's magnetosphere. The input model parameters for the inbound Pioneer 11 pass: $R_{S S}=17.5 R_{S} ; \quad l_{o}=12.5 R_{S} ; \quad l_{i}=6.5 R_{S} ; \quad \Psi=0.95^{\circ} ; \quad R_{t}=14 R_{S}$; $B_{t}=8.7 \mathrm{nT} ; B_{l o}=3.62 \mathrm{nT}$.

From Fig. 1 it follows that more accurate modeling of the Saturn ring current should be done in the future, in particular, probably another dependence of the ring current strength on the radial distance $r$ should be considered.

In the inner magnetosphere, $r \leq 5.8 R_{S}$, the main field is created by the sources inside the planet (magnetospheric external sources give there $\sim 0.1$ of the total field at $r \sim 5.8 R_{S}$, and $\sim 0.001$ of the total field at the closest approach of the Pioneer 11, respectively). The internal Saturn's field is relatively constant. Thus, it is possible to combine measurements taken along many orbits to produce the internal field model. Here we used the SPV model (Davis and Smith, 1990) constructed from the Pioneer and Voyagers data.

We should emphasize that the suggested method differs in principle from methods based on a least-squares fitting (e.g. Giampieri and Dougherty, 2004). Using analytical formulas allows us to obtain the approximate expressions for the field's $z$ component along the Saturn-Sun line, which makes it possible to receive simple equations for the determination of the paraboloid model parameters. The obtained results can be considered as a first step, further allowing a more accurate definition. However, even now we received rather good precision of the fulfilled computation. Figure 2 shows the noon-midnight cross section of Saturn's magnetosphere, constructed using the obtained set of the input model parameters.

Paraboloid magnetospheric models for different magnetized planets (Earth, Jupiter, and Saturn) have been developed using a module structure (magnetic field of each current system was calculated separately). The typical input magnetospheric model parameters for these planets are the following. For the Earth, the distance to the subsolar magnetopause is $R_{s e}=10 R_{E}$; the distance to the inner edge of the tail current sheet is $R_{t}=8 R_{E} ; B_{t} / \alpha_{0}=24.84 \mathrm{nT}$ is the tail current system magnetic field at the inner edge of the tail current sheet $\left(B_{t}=40 \mathrm{nT}, \alpha_{0}=1.61\right)$, where $R_{E}$ is the Earth radius (e.g. Alexeev, 1986; Alexeev et al., 1996)). For Jupiter (for the Ulysses epoch), the distance to the subsolar magnetopause is $R_{s j} \sim 100 R_{J}$; the distance to the inner edge of the tail current sheet is $R_{t} \sim 65 R_{J} ; B_{t} / \alpha_{0}=-0.33 \mathrm{nT}$ is the tail current system magnetic field at the inner edge of the tail current sheet ( $\left.B_{t} \sim-0.5 \mathrm{nT}, \alpha_{0}=1.52\right)$; the distances to the outer and inner edges of the magnetodisk are $R_{D 1} \sim 92 R_{J}$ and $R_{D 2} \sim 65 R_{J}$, respectively; the current disk magnetic field strength in the outer edge of the current disc is $B_{D C}=2.5 \mathrm{nT}$, where $R_{J}$ is Jupiter's radius (Belenkaya, 2003, 2004; Alexeev and Belenkaya, 2005). For Saturn (for the Pioneer 11 inbound flyby), the distance to the subsolar magnetopause is $R_{s s}=17.5 R_{S}$; the distance to the inner edge of the tail current sheet is $R_{t}=14 R_{s} ; B_{t} / \alpha_{0}=-5.4 \mathrm{nT}$ is the tail current system magnetic field at the inner edge of the tail current sheet ( $\left.B_{t}=-8.69 \mathrm{nT}, \alpha_{0}=1.61\right)$; the distances to the outer and inner edges of the ring current are $l_{o}=12.5 R_{S}$ and $l_{i}=6.5 R_{S}$, respectively; the ring current magnetic field strength in the outer edge of the ring current is $B_{l o}=3.62 \mathrm{nT}$ ( $R_{S}$ is Saturn's radius).

\section{Conclusions}

Paraboloid magnetospheric models for different magnetized planets (Earth, Jupiter, and Saturn) have been developed using a module structure (magnetic field of each current system was calculated separately). Due to the planet's rapid rotation and the existence of the internal magnetospheric plasma sources, the magnetodisk and the ring current are created in the Jovian and kronian magnetospheres, respectively.

Saturn's magnetosphere is intermediate to those of Earth and Jupiter. On the basis of the earlier constructed terrestrial and Jovian models, a paraboloid Saturnian magnetospheric model is developed. This model of the kronian magnetospheric magnetic field includes a planet's field, the ring and tail currents fields, the magnetopause shielding currents field, and magnetic field penetrated from the solar wind. Some of the magnetic field sources included in this model were 
not considered previously; for example, we incorporated the magnetotail and magnetopause currents contribution, which should improve Saturn's global magnetospheric model. The method of the model parameters' determination is demonstrated by using the approximate formulas simulating the magnetic fields of different magnetospheric current systems along the Saturn-Sun line. On the example of the inbound Pioneer 11 data, all model parameters are determined. The obtained set of the input model parameters was used for computation of the magnetospheric magnetic field along the inbound Pioneer 11 trajectory. Comparison with measurements showed good accordance, nevertheless, further tuning of the input parameters will allow one to refine them to fit the better available observations.

The paraboloid models constructed previously for the Earth (Alexeev, 1978, 1986) and Jupiter (Belenkaya, 2004; Alexeev and Belenkaya, 2005) allowed us to receive significant results. The terrestrial model was successfully applied, for example, for the description of magnetic storms (Alexeev et al., 1996), for explanation of the magnetospheric responses to coronal mass ejection encounters under specific conditions in the interplanetary magnetic field (Clauer et al., 2001; Belenkaya et al., 2004), for simulation of theta-aurora (Blomberg et al., 2005), etc. For Jupiter, the paraboloid model allows one to explain the existence of anti-corotation flows in the morning sector of the equatorial magnetosphere observed by Ulysses, and the absence of such flows during the Voyager flybys (Belenkaya, 2003, 2004); the model calculations yielded the location of the main auroral oval corresponding to the maximum of the field-aligned potential electric drops (Belenkaya, 2003, 2004; Alexeev and Belenkaya, 2005). Now we modified the paraboloid model for Saturn. This model is an effective instrument, allowing one to analyze the planet's environment (for example, it explained the dependence of Saturn's aurora in the January 2004 events on the interplanetary magnetic field, Belenkaya et al., 2006).

For successful application of the paraboloid model, it is necessary to determine the model's parameters, which are very variable dependent on the conditions in the solar wind and inside the kronian magnetosphere. These dependences are described in the present paper, and a method of parameter determination is demonstrated for the first flight to Saturn performed by the Pioneer 11 .

Acknowledgements. This work was partially supported by the INTAS Grant No 03-51-3922 and by the RFBR Grants 04-05-64396 and 05-05-64435. We are grateful to the team providing the data of Helium Vector Magnetometer for the Pioneer 11 which we obtained using the NASA's Planetary Data System (http://pds.jpl.nasa.gov/).

Topical Editor I. A. Daglis thanks two referees for their help in evaluating this paper.

\section{References}

Acuña, M. H., Ness, N. F., and Connerney, J. E. P.: The magnetic field of Saturn: further studies of the Pioneer 11 observations, J. Geophysical Res., 85, A11, 5675-5678, 1980.

Alexeev, I. I.: Regular magnetic field in the Earth's magnetosphere, Geomagn. and Aeronom. (in Russian), 18, 656-665, 1978.

Alexeev, I. I.: The penetration of interplanetary magnetic and electric fields into the magnetosphere, J. Geomag. Geoelectr., 38, 1199-1221, 1986.

Alexeev, I. I. and Belenkaya E. S.: Modeling of the Jovian magnetosphere, Ann. Geophys., 23, 809-826, 2005.

Alexeev, I. I., Belenkaya, E. S., Kalegaev, V. V., Feldstein, Y. I., and Grafe A.: Magnetic storms and magnetotail currents, J. Geophys. Res., 101, 7737-7747, 1996.

Alexeev, I. I., Belenkaya, E. S., and Clauer, C. R.: A model of region 1 field-aligned currents dependent on ionospheric conductivity and solar wind parameters, J. Geophys. Res., 105, 21119 $21127,2000$.

Alexeev, I. I., Kalegaev, V. V., Belenkaya, E. S., Bobrovnikov, S. Y., Feldstein, Ya. I., and Gromova, L. I.: Dynamic model of the magnetosphere: Case study for January 9-12, 1997, J. Geophysical Res., 106, 25 683-25 694, 2001.

Alexeev, I. I., Belenkaya, E. S., Bobrovnikov, S. Yu., and Kalegaev, V. V.: Modelling of the electromagnetic field in the interplanetary space and in the Earth's magnetosphere, Space Sci. Rev., 107, 726, 2003

Alexeev, I. I., Kalegaev, V. V., Belenkaya, E. S., Bobrovnikov, S. Yu., Bunce, E. J., Cowley, S. W. H., and Nichols, J. D.: A global magnetic model of Saturn's magnetosphere and a comparison with Cassini SOI data, Geophys. Res. Lett., 33, L08101, doi:10.1029/2006GL025896, 2006.

Bastian, T. S., Chenette, D. L., and Simpson, J. A.: Charged particle anisotropies in Saturn's magnetosphere, J. Geophysical Res., 85, No A11, 5763-5771, 1980.

Behannon, K. W., Connerney, J. E. P., and Ness, N. F.: Saturn's magnetic tail: Structure and dynamics, Nature, 292, 753-755, 1981.

Behannon, K. W., Lepping, R. P., and Ness, N. F.: Structure and dynamics of Saturn's outer magnetosphere and boundary regions, J. Geophysical Res., 88, A11, 8791-8800, 1983.

Belenkaya, E. S.: Peculiarities of the interaction between the solar wind magnetic field with the terrestrial and Jovian magnetospheres, Geomagn. and Aeronom (in Russian), 43, No 2, 17-4$182,2003$.

Belenkaya, E. S.: The Jovian magnetospheric magnetic and electric fields: effects of the interplanetary magnetic field, Planet. Space Sci., 52, No 5-6, 499-511, 2004.

Belenkaya, E. S.: Transition current systems in the terrestrial and kronian magnetospheres, Geomagn. and Aeronom (in Russian), in press, 2006.

Belenkaya, E. S., Alexeev, I. I., and Clauer, C. R.: Field-aligned current distribution in the transition current system, J. Geophys. Res., 109, A11207, doi:10.1029/2004JA010484, 2004.

Belenkaya, E. S., Bobrovnikov, S. Y., Alexeev, I. I., Kalegaev, V. V., and Cowley, S. W. H.: A model of Jupiter's magnetospheric magnetic field with variable magnetopause flaring, Planet. Space Sci. 53, No 9, 863-872, 2005. 
Belenkaya, E. S., Cowley, S. W. H., and Alexeev, I. I.: Saturn's aurora in the January 2004 events, Ann. Geophys., 24, in press, 2006.

Blomberg, L. G., Cumnock, J. A., Alexeev, I. I., Belenkaya, E. S., Bobrovnikov, S. Yu., and Kalegaev, V. V.: Transpolar aurora: time evolution, associated convection patterns, and a possible cause, Ann. Geophys., 23, 1917-1930, 2005.

Bunce, E. J. and Cowley, S. W. H.: A note on the ring current in Saturn's magnetosphere: Comparison of magnetic data during Pioneer-11 and Voyager-1 and -2 fly-bys, Ann. Geophys., 21, 661-669, 2003.

Bunce, E. J., Cowley, S. W. H., and Wild J. A.: Azimuthal magnetic fields in Saturn's magnetosphere: Effects associated with plasma sub-corotation and magnetopause-tail current system, Ann. Geophys., 21, 1709-1722, 2003.

Clauer Jr., C. R., Alexeev, I. I., Belenkaya, E. S., and Baker, J. B.: Special features of the September 24-27, 1998 storm during high solar wind dynamic pressure and northward interplanetary magnetic field, J. Geophysical Res., 106, A11, 25695-25712, 2001.

Connerney, J. E. P., Acuña, M. H., and Ness, N. F.: Modeling the Jovian current sheet and inner magnetosphere, J. Geophysical Res., 86, 8370-8384, 1981a.

Connerney, J. E. P., Acuña, M. H., and Ness, N. F.: Saturn's ring current and inner magnetosphere, Nature, 292, 724-726, $1981 \mathrm{~b}$.

Connerney, J. E. P., Ness, N. F., and Acuña, M. H.: Zonal harmonic model of Saturn's magnetic field from Voyager 1 and 2 observations, Nature, 298, 44-46, 1982.

Connerney, J. E. P., Acuña, M. H., and Ness, N. F.: Currents in Saturn's magnetosphere, J. Geophysical Res., 88, No A11, 87798789, 1983.

Cowley, S. W. H. and Bunce, E. J.: Corotation driven magnetosphere-ionosphere coupling currents in Saturn's magnetosphere and their relation to the auroras, Ann. Geophys., 21, 1691-1707, 2003.

Cowley, S. W. H., Bunce, E. J., and Prange, R.: Saturn's polar ionospheric flows and their relation to the main auroral oval, Ann. Geophys., 22, 1379-1394, 2004.

Cumnock, J. A., Blomberg, L. G., Alexeev, I. I., Belenkaya, E. S., Bobrovnikov, S. Yu., and Kalegaev, V. V.: Simultaneous polar aurorae and modelled convection patterns in both hemispheres, Adv. Space Res., in press, 2005.

Davis Jr., L. and Smith, E. J.: New models of Saturn's magnetic field using Pioneer 11 vector helium magnetometer data, J. Geophysical Res., 91, A2, 1373-1380, 1986.

Davis Jr., L. and Smith, E. J.: A model of Saturn's magnetic field based all available data, J. Geophysical Res., 95, A9, $15257-$ 15 261, 1990.

Frank, L. A., Burek, B. G., Ackerson, K. L., Wolfe, J. H., Mihalov, J. D.: Plasmas in Saturn's magnetosphere, J. Geophysical Res., 85, A11, 5695-5708, 1980.
Galopeau, P. and Lecacheux, A.: Variations of Saturn's radio rotation period measured at kilometer wavelengths, J. Geophysical Res. 105, A6, 13 089-13 101, 2000.

Giampieri, G. and Dougherty, M. K.: Modelling of the ring current in Saturn's magnetosphere, Ann. Geophys., 22, 653-659, 2004.

Hendricks, S., Neubauer, F. M., Dougherty, M. K., Achilleos, N., and Russell, C. T.: Variability in Saturn's bow shock and magnetopause from Pioneer and Voyager: Probabilistic predictions and initial observations by Cassini, Geophys. Res. Lett., 32, L20S08, doi:10.1029/2005GL022569, 2005.

Hill, T. W.: The Jovian oval, J. Geophysical Res., 106, A5, 81018107, 2001.

Maclennan, C. G., Lanzerotti, L. J., Krimigis S. M., and Lepping, R. P.: Low-energy particles at the bow shock, magnetopause, and outer magnetosphere of Saturn, J. Geophysical Res., 88, A11, 8817-8830, 1983.

Maurice, S. and Engel, I. M.: Idealized Saturn magnetosphere shape and field, J. Geophysical Res., 100, 17 143-17 151, 1995.

Maurice, S., Engel, I. M., Blanc, M., and Skubis M.: Geometry of Saturn's magnetopause model, J. Geophysical Res., 101. A12, 27 053-27 059, 1996.

McDonald, F. B., Schardt, A. W., and Trainor, J. H.: If you've seen one magnetosphere, you haven't seen them all: Energetic particle observations in the Saturn magnetosphere, J. Geophysical Res., 85, A11, 5813-5830, 1980.

Ness, N. F., Acuña, M. H., Lepping, R. P., Connerney, J. E. P., Behannon, K. W., Burlaga, L. F., and Neubauer, F.: Magnetic field studies by Voyager 1: Preliminary results at Saturn, Science, 212, 211-217, 1981.

Richardson, J. D.: Thermal ions at Saturn: Plasma parameters and implications, J. Geophysical Res., 91, A2, 1381-1389, 1986.

Sauer, J., Mauk, B. H., Kassner, A., and Neubauer, F. M.: A model for the azimuthal plasma velocity in Saturn's magnetosphere, J. Geophysical Res., 109, A05217, doi:10.1029/2003JA010207, 2004.

Sittler Jr., E. C., Oglive, K. W., and Scudder, J. D.: Survey of lowenergy plasma electrons in Saturn's magnetosphere: Voyagers 1 and 2, J. Geophysical Res., 88, 8847-8870, 1983.

Smith, E. J., Davis, L., Jones, D. E., Coleman, P. J., Colburn, D. S. Dyal, P., and Sonett, C. P.: Saturn's magnetosphere and its interaction with the solar wind, J. Geophysical Res., 85, A11, 56555674, 1980.

Tsyganenko, N. A.: Modeling the Earth's magnetospheric magnetic field confined within a realistic magnetopause, J. Geophysical Res., 100, 5599-5612, 1995.

Tsyganenko, N. A.: Effects of the solar wind conditions on the global magnetospheric configurations as deduced from databased field models, in Proceedings of 3rd International Conference on Substorms, Versailles, France, ESA SP-389, Eur. Space Agency, Paris, 181-185, 1996. 